Michael S. Debus

IT University of Copenhagen

\title{
Video Game Navigation: A Classification System for Navigational Acts
}

\section{Introduction}

The young field of game studies has produced a multitude of works regarding matters of space and time in video games (e.g. Gazzard 2009a; Günzel 2010; Nitsche 2008; Tychsen \& Hitchens 2009; Zagal \& Mateas 2007). Interestingly, these discussions are limited to the environments in which players play. Others, which actually examine videogame navigation, include socio-cultural practices of navigation (e.g. Chesher 2012), or the players' experience of the gameworld through navigation (e.g. Flynn 2008). The question of how they traverse space and time has been neglected for the biggest part.

Several studies indicate the importance of navigation for the player experience and the game space. Van Driel and Bidarra state that " $[w]$ hile the game world remains the same, an increase of abilities makes the AI perceive a richer world with more paths" (2009, p. 153). While they are concerned with developing a better method for AIs to navigate and understand space, this statement can easily be applied to human players as well. Alison Gazzard states that "[o]ther vehicles increase this speed and therefore can significantly alter our understanding of the path and the relationship we have with it” (2009a, p. 40). Bernadette Flynn connects Markku Eskelinen's observation that the user function in games is primarily a configurational rather than an interpretational one (Eskelinen 2001) with the act of navigation (Flynn 2008, p. 137). Even earlier, she argues for the importance of navigation in videogames as "[...] it is only through navigation that gameplay acquires a language and this language then operates at the level of a central organizing device" (Flynn 2003, p. 8). 
Navigation lies at the core of many, especially contemporary, videogames and has an important impact on the player's experience of the game. Curiously, even though video game navigation's socio-cultural contexts (e.g. Flynn 2003; Chesher 2012), and the player's experience of space through it (Flynn 2008) have been discussed, what is still missing is an examination of the navigation itself. Flynn's (2008) phenomenologically inspired discussion includes, for example, the navigation of Myst (Cyan 1993) and Half Life (Valve 1998), without further examining whether the acts of navigation in these games are different. As Espen Aarseth argues, every videogame study should be supported by ontological research (2014). Such an ontology, and with it a clearer terminology, is missing for navigation in videogames. This paper examines different types of navigational acts and demarcates them from one another, arriving at a classification system that will support future inquiries into the topic of videogame navigation.

A navigational act is any kind of movement, relocation, or teleportation that transfers a player's avatar from one location to another. The term 'navigational act' was chosen, as other possibilities, such as 'process', did not account for the specificity of this investigation. A 'process', as defined by the Oxford English Dictionary, is “a series of actions or steps taken in order to achieve a particular end". The problem here is, as will be clearer later, that the definition of relocation as "a series of actions" is questionable. To ensure the specific focus on how the player navigates the gameworld and the limitation to the smallest 'navigational unit', the term 'navigational act' was chosen. The classification of these acts was developed through the analysis of existing theoretical works as well as the critical play of multiple games.

Michael Nitsche distinguishes five planes of video game spaces (2008, p. 15): the rule-based space, mediated space, fictional space, play space, and social space. The games, and thereby the problem of a missing classification of navigational acts, were examined on Nitsche's third plane, mediated space, as the visual (and auditive) presentation of the game's code to the player. Additionally, influences by the game's rules and code (first plane) and on the player's fiction that is developed in her mind (third plane) will be discussed occasionally, when appropriate and fruitful.

First, an overview of important, relevant work in the field of game studies will be presented and some terms that are necessary for the later classification will be developed. After this, the classification model will be explained in a step-by-step manner, including examples from analyzed video games and discussions of pre-existing classifications. 


\section{Videogame Spaces}

While it has been argued earlier that studies tend to focus on what we navigate in instead of how we navigate in it, it is indeed important to have a basic understanding of the what before we can discuss the how. One of these environments is game space, which has been examined in game studies before. In their approach towards a game typology, Christian Elverdam and Espen Aarseth (2007) came up with three subcategories for the metacategory virtual space: Perspective, Positioning, and Environmental Dynamics. In the context of the present work the subcategory of Perspective is negligible, as it describes the player's view onto the gameworld, not the game space itself. The same goes for the category Environmental Dynamics, as it refers to the possibilities of alterations to the gameworld. However, the subcategory of Positioning will be further discussed.

As described by the authors, the positioning of the player inside the virtual space can be either absolute or relative (Elverdam \& Aarseth 2007, p. 7). In the first case, the player is only able to navigate between locations that are predetermined by the game. A relative position, on the other hand, means that the player can navigate freely inside the virtual space and her position would be described depending on other objects or players inside the virtual space. The difference between the two is briefly described thusly: "The pawn stands on C3" in Chess (absolute) versus "I am next to the three green boxes on bomb spot B" in Counter-Strike (Valve Corporation 2000) (relative). It is interesting to see the difference between this typology and the one it is derived from, an earlier approach towards a game typology by Aarseth et al. in 2003. Here the authors state that "A game's topography can be either geometrical, with continuous freedom of movement, or topological, giving the player only discrete, non-overlapping positions to move between" (Aarseth et al. 2003, pp. 49-50).

At first, the shift of focus between the two typologies should be mentioned. In the chronologically earlier typology, Aarseth et al. (2003) actually tried to categorize the video game space itself, whereas in the latter, Elverdam and Aarseth (2007) used a description of the player's positioning inside the gameworld. What is more important are the similarities between the two (one is derived from the other after all): the dyadic relation of absolute and relative (Elverdam \& Aarseth 2007, p. 7), and topological and geometrical (Aarseth et al. 2003, pp. 49-50), where the former describes discrete restriction and the latter depicts continuous freedom.

A similar distinction was made by Deleuze and Guattari in 1987. In their book A Thousand Plateaus, they describe space as smooth or striated (Deuleuze \& Guattari 1987, p. 474). Here smooth describes a continuous space, such as a desert or a sea, just 
like what was called geometrical by Aarseth et al. (2003). Striated space is similar to what Aarseth et al. called topological: divided areas existing next to each other, and the player can only be inside one or the other.

This dichotomy is a recurring theme in concepts developed in game studies and the more general discussion of space. As mentioned before, Aarseth et al. (2003) describe geometrical and topological spaces and Elverdam \& Aarseth (2007) refine these into an absolute or relative positioning by the player. Furthermore, Michael Nitsche (2008, p. 182) adopts Deleuze \& Guattari's (1987) terms to describe the change of space in Doom, when switching between the first-person view and a bird's-eye view onto a two-dimensional map.

These differences, especially the determining character of striated space, will have important implications for the classification of navigational acts.

\section{Time in Videogames}

Videogames offer the possibility to navigate not only space but also time. Therefore, it is equally important to briefly examine existing approaches towards game time analysis. Zagal and Mateas' (2007) approach follows a reductionist tradition of understanding time by analyzing time frames through the relation of events in the real world, the gameworld, their organization in coordination frames and the fictive time frames connoted with them. Tychsen and Hitchens (2009), without explicitly stating it, include the platonist view of time into their model, by considering time's continuous flow in the player's engine, the server, and the real world. While these approaches can be subsumed under what Michael Nitsche calls “formalist approaches" (2007, p. 145), we should not forget the subjective role of the player, as added by Tychsen and Hitchens in their "perceived representation of time" (2009), and more thoroughly discussed by Nitsche (2007). However, it is important to note that time - just like space - can be distinguished into topological and geometrical systems. Zagal and Mateas' "coordination time" (2007), for example, describes rounds or turns in games, which are of a similar 'either-or' nature as the topological space in games. The category "server time", on the contrary, describes the continuous (geometrical) flow of time on the server.

These models show that the matter of time in video games is complex. Considering the classification of temporal navigation, the application of one of these models - or even all of them - is unpractical. Such an application would have to include descriptions and analyses of not only the navigation inside of each frame or representation of time, but also how the different types relate to each other between frames or represen- 
tations of time. Due to the complexity of such a discussion, it is fruitful to use a more simplified model, which will be developed below.

This model draws from Bordwell and Thompson's terms "story" and "plot" (2008) and Gordon Calleja’s term "alterbiography” (2009). In the present, simplified model the first distinction is made between prescribed story and prescribed plot. The prescribed story describes the game's events in their original, scripted order, while the prescribed plot describes the events as presented to the player. Following Bordwell and Thompson, the plot is "[...] everything visibly and audibly present in the film before us" (2008, p. 16). Opposed to this, the prescribed story further includes events that are not directly visible to the player, but may be part of the game's lore in general. In this sense, the prescribed story cannot be seen as segmented, as it includes all theoretical events from the 'birth' of the game's universe until its last (or future) events. In other words, the game's prescribed story is a continuous stream of events (is geometrical) and the prescribed plot consists of chunks (is topological). Whether these chunks are in chronological order is unimportant, as opposed to the fact that the game forces the player to play chunks of events. The last term that is necessary for the classification's development is alterbiography of events. Derived from Gordon Calleja's "alterbiography" (2009), which describes each player's own 'story' while playing the game, the term alterbiography of events describes the order of events as they occurred during each individual play-through. While these three terms bring a certain inaccuracy with them, they enable us to describe and understand temporal navigation on a basic level first, before discussing it within the frame of more complex models, such as the ones described above.

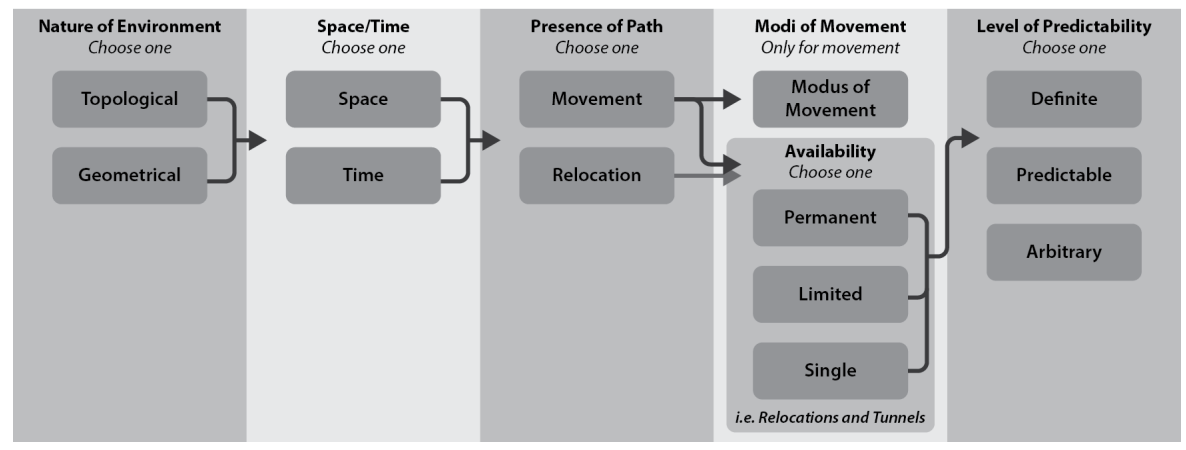

Figure 1. Step-by-Step classification of navigational acts

\section{The Typology}

Drawing from the previously described related works, the following sections will develop the classification for navigational acts. Each section consists of one of the typology's five dimensions and the complete model will be followed by exemplary applications of it. 


\section{Nature of Environment}

As discussed earlier, the main difference in video game spaces and time are topological and geometrical (Aarseth et al. 2003), striated and smooth (Deleuze \& Guattari 1987), or absolute and relative (Elverdam \& Aarseth 2007) representations of space and time. Stefan Günzel describes the difference between the video game space categories (topological and geometrical) established by Aarseth et. al (2003) as "the difference between continuous movement and discrete movement" (Günzel 2010, p. 174). As he points out, the navigational options in a geometrical (continuous) environment are different from the options a topological (discrete) one offers. Therefore, we have to distinguish between geometrical and topological navigation on the first, superordinate level.

\section{Navigation in Space or Time}

After distinguishing between these two overall categories, the second level differentiates between spatial and temporal navigation. Some video games give us the interesting possibility to not only navigate virtual spaces but also time. Unlike in real life, books, or movies, in video games we can actively rewind time to replay a situation in a different way than before, fast forward it, or navigate to completely different temporal locations. The distinction between spatial and temporal navigation is necessary, as the two do not necessarily occur together.

It has to be noted that certain types of navigation (especially movement) are closely linked to a simultaneous navigation in time, as time automatically passes. However, this only supports the necessity for splitting the spatial and temporal aspects of navigational acts: a spatial relocation, for example, can be temporal movement. However, for a detailed and accurate analysis of navigational acts a differentiation between their temporal and spatial aspects can be useful.

\section{Presence of Path}

On this third level a distinction between relocations and movement is made. To exemplify this distinction, it is useful to briefly discuss the relationship of time and movement. As discussed earlier, video games can have multiple time frames (Zagal \& Mateas 2007) or representations of time (Tychsen \& Hitchens 2009). This shows that, if we include time into the definition of movement, we would always have to specify what time we are referring to when analyzing temporal navigation. In videogames, the gameworld time (Zagal \& Mateas 2007) does not necessarily proceed while the character is moving. This becomes even more complicated when we include several players or non-player characters (NPCs), who have their own histories or play times. The way to 
solve this problem would be to specify the time in the definition of movement as our real time. A second problem remains, though: how do we categorize movement of, for example, very fast avatars or characters?

In the fighting game Dragon Ball XenoVerse (Dimps 2015) the player is placed into the world of the Dragon Ball saga. By pressing the buttons 'L2' and 'A' just before the opponent lands a hit, the player uses two of her stamina points to avoid the hit and to reappear behind the opponent. This would generally be considered a relocation. However, fans of the saga would object to this classification, as the move is explained by the characters' possibility to move inhumanly fast. The passing of time is very subjective: while humans experience Son Goku's move as instantaneous, for him there is enough time to move behind his enemy.

To exclude the subjectivity of time, movement has to be understood as transitioning between two locations through a path of adjacent locations in between them. In geometrical navigation, there are nearly infinite possible locations between the start and end locations of movement. Through the absolute nature of navigation in topological systems, these locations are easier to count. Relocation, then, is the act of transitioning between start and end location without passing a path of adjacent locations. Depending on whether we take the game mechanics or the game's lore as the basis for discussion, the dodge in Dragon Ball XenoVerse is either movement (lore) or relocation (mechanics).
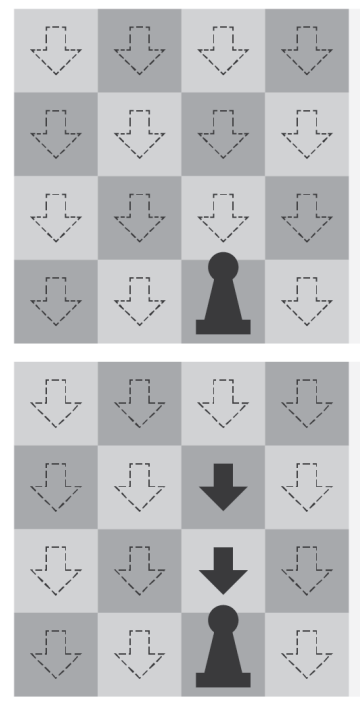

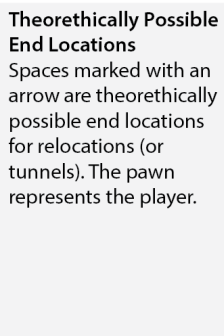

Predictable End Location

The theoretically possible end locations are limited to a certain amount of actually possible end locations. The end location can be any of the actually possible end locations.

Figure 2. Level of Predictability

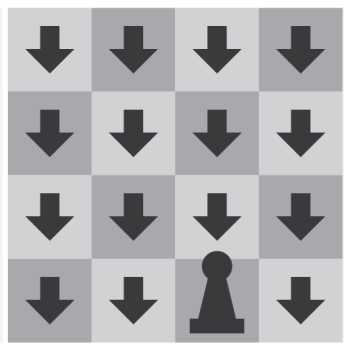

Arbitrary End Location The actual end location can be any of the theoretically possible end locations.

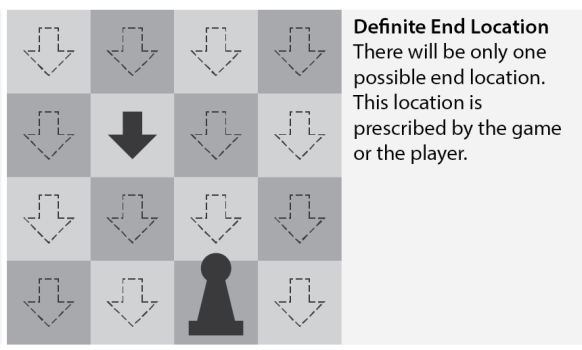


Following this distinction, using the Citadel of Time in The Legend of Zelda: Ocarina of Time (Nintendo EAD 1998) is a temporal move in the game's prescribed plot (topological environment) and alterbiography of events (geometrical environment), but a temporal relocation in the game's prescribed story (geometrical environment).

Before the typology's fourth level can be described and discussed, it is important to differentiate between tunnels as subcategories of movement, and relocations as acts of navigation that are separate from tunnels. This is because they have identical subcategories on the typology's fourth and fifth level, even though they are two different navigational acts. While relocations were described as instantaneously transitioning from one location to another, tunnels enable the player to do something similar through continuous movement. To understand tunnels it is useful to examine the difference between teleporters in Torchlight II (Runic Games 2012) and the portals in Portal 2 (Valve Corporation 2011). In Torchlight II the teleporters are installed devices through which the player can instantaneously relocate to a nearby, isolated area. The portal gun in Portal 2 enables the player to create portals on surfaces in the game, which function as entrance and exit for a tunnel. The main difference is not the possibility of creating the tunnel. More important is the fact that the player navigates the tunnels in Portal 2 through continuous movement, while the relocation in Torchlight II is instantaneous. Following Stefan Günzel's argument (2010), one can also describe the act of navigation in cases of relocation as discrete, while navigation through a tunnel is continuous. This difference enables the player's avatar in Portal 2 to be partially on both sides of the portal at the same time, while, in instances of relocation, the avatar's position is exclusively on one side or the other.

By creating a tunnel in Portal 2 the player alters the (game) space to create a shortcut between two locations in the level. Therefore, tunneling is the act of continuously moving through altered (game) space. Technically, one could further distinguish between tunnels which alter the game space in a more general sense and those which alter the space itself. The portals in Portal 2 create a direct shortcut between two locations in a level that are originally far away from each other. To understand this, we could argue that the portal gun bends space itself in certain locations to turn them into adjacent ones, which is very similar to the explanation of wormholes or warp engines in science fiction. While this is a manipulation of space itself, digging a tunnel through a mountain in a gameworld is also tunneling. However, each act of tunneling through space - in the science fiction sense - is also an alteration of the gameworld. Therefore, this difference will be neglected in the present typology. 
The fact that navigating through tunnels is a continuous act of navigation shows another difference between them and relocations. As Alison Gazzard observes, " $[t]$ he warp turns paths experienced by the player into fixed 'tracks', where navigational control is removed whilst in the warp sequence [...]" (2009b, p. 1). While this is true for relocations in the present typology, it is not for tunnels. The player loses control over the avatar during the instantaneous relocations, whereas tunnels do not remove control completely, only restrict it.

\section{Availability and modi of movement}

The typology's fourth level describes two things. First, 'normal' movement includes all modi of movement such as walking, running, crawling, jumping, etc., as well as rewinding or forwarding time. Therefore, the final classification of 'running over a mountain' in Zelda will be seen here as geometrical, spatial movement in the modus running. The modi of 'normal' movement are too diverse to list them all, and therefore the reader has to refer to common language to describe them.

Contrary to this, as indicated through Gazzard's work on warps (2009a, 2009b), a further classification of the tunnels and relocations is possible and useful. The concept of warps subsumes the here described tunnels and relocations. Therefore, one option would be to adopt Gazzard's classification into the present typology as it is. However, especially because Gazzard was concerned with the nature of the path and not the navigational act itself, there are certain problems with the direct application of her warp classification to navigational acts. In addition to that, her classification poses some minor, inherent problems. As direct adoption is not possible, it is necessary to first describe her model and discuss the aforementioned problems. Following this discussion, the model will be improved and adapted into the present typology.

Gazzard distinguishes between three overall categories: Jump warps, return warps and portals (2009b p. 4). For her, jump warps are unidirectional (ibid.), meaning the player is at location A first and then at location B, without the possibility of navigating back the same way. She further distinguishes between visible and aleatoric jumps. In the former case, the warp's start and end point are visible to the player, while aleatoric jumps provide the player with no visibility of the jump's end point.

The second category, i.e. return warps, enable the player to travel back the same way and are therefore bidirectional (Gazzard 2009b, p. 5). Similar to the distinction of visible jumps, Gazzard describes two subcategories of return warps. Return to previous is a warp from location A to B and then back to A. During a return to other warp, the player does not return to her original start location, but to a different one: location 
C. The town portals in Diablo 3 (Blizzard Entertainment 2012), for example, are return to previous warp devices, while examples of return to other warps are hard to find, as their arbitrary mechanics would likely confuse players (Gazzard 2009a, pp. 153-154).

Gazzard's model's first layer distinguishes warps by 'direction'. Jump warps are defined by being unidirectional, while return warps and portals are bidirectional. The second layer classifies by the 'visibility' of start and end points. It is actually here where portals are distinguished from jump and return warps. This means that the split into three types of warps on Gazzard's first layer is executed by applying the criteria of layer one and two to portals. Strictly following these distinctive criteria, portals should be a subcategory of return warps, as they offer a bidirectional use for the player. On the second layer, then, they would be a separate category through their permanent visibility of both their start and end points.

Furthermore, her example for the distinction between visible jumps and aleatoric jumps are the "inadvertent warps" (Gazzard 2009b, p. 4) of Super Mario Bros. (Nintendo R\&D4 1985). The designers hid tubes in the game, through which the player can warp to more advanced levels. For Gazzard, these tubes are aleatoric warps because "[...] the end point cannot be seen as the new path is determined by the games system" (Gazzard 2009b, p. 4). In fact, the tubes in Mario Bros have a number displayed above them, indicating the level they lead to. Additionally, the tubes always take the player to the same location at the start of the level that is indicated by the number. Therefore, the warp's end location is not actually visible, but the player could know where she will arrive if she uses the tube.

This leads us to a problem with the term aleatoric. It results from the adoption of Roger Caillois' alea, which describes “[...] all games that are based on a decision independent of the player, an outcome over which he has no control [...]" (Caillois 1961, p. 17). In fact, Callois describes alea not only as the player's powerlessness to determine the game's outcome, as the aforementioned quote continues: “[...] and in which winning is the result of fate rather than triumphing over an adversary" (ibid.). Further he states that "[p]erfect examples of this type are provided by the games of dice, roulette, heads or tails, baccara, lotteries, etc." (ibid.). Callois' alea therefore does not only limit the player's influence on the outcome, it also ascribes this outcome to fate or chance. To be clear: The derivation of Caillois' term alea seems problematic due to the exclusion of arbitrariness in Gazzard's concept. Thus, the present model will not use the player's powerlessness over the outcome of the warp as criterion for classification, but the navigational act's arbitrariness - or predictability - to include both parts of Caillois' alea.

Alison Gazzard's classification of warp devices is an important pioneering work in the field of video game navigation, and it is fruitful to adapt her model into the present 
classification system. As she was concerned with the path's nature and not the navigational act itself, and due to the discussion in the last paragraphs, her model will not be adopted, but adapted in the following.

Finally arriving at the present typology's fourth layer of availability, Gazzard's model's first layer has to be adjusted not by distinguishing between the direction (unidirectional, bidirectional) of warps, but by their availability. The three categories here are single use, limited use and permanent use. Single use warps are warps that can be used only one time and, therefore, are similar to but not the same as "unidirectional tracks" (Gazzard 2009b, p. 3). Considering Gazzard's model, when the player uses a unidirectional track, she is not able to use the same warp to move back again. In a way, the warp is only available to the player once, which is where the two models overlap. However, it is also possible that the player uses the same warp again, after moving back to its start point through different means. This is where the criteria of direction (Gazzard) and availability (present typology) differ. Limited use means that the warp can be used only a certain amount of times, unlike permanent use warps, which are available for the player as often as she wants. Examples are Zeratul's 'Blink' ability in Heroes of the Storm (Blizzard Entertainment 2015) for single use warps, Pikachu's 'up + B' move in Super Smash Bros. (HAL Laboratory 1999) for limited use warps, and the circles of light in The Talos Principle (Croteam 2014) for permanent use warps.

The advantage of this distinction is that we can distinguish between "return warps" and "portals" (Gazzard 2009b) on the first level, without adding the criteria of the second level to "portals" as well. By adapting Gazzard's approach to further classify warps by their start and end locations, they will be divided by their predictability into definite, predictable and arbitrary warps.

\section{Level of Predictability}

For the description of start and end locations the terms 'theoretically possible' and 'actually possible' (end) locations will be used. All locations which are accessible through a certain spell or ability will be referred to as 'theoretically possible (end) locations'. In other words, a hole in the ground is not a theoretically possible end location, while the rest of the area is. 'Actually possible (end) location' will refer to the possible end locations from one specific start location.

The term arbitrary is derived from Gazzard's term "aleatoric" (2009b, p. 4). However, as she focuses on the player's powerlessness to determine the outcome of the warp but not Caillois' inclusion of chance into the term, it is fruitful to use the different term arbitrary here. The end points of these warps are not only determined by the 
game system but they are completely arbitrary. Definite warps, on the contrary, have a specific start and end location. This can be either a location coded into the game, which is always the same, or a location chosen by the player. Predictable warps are in between definite and arbitrary warps. Here the player is able to determine the end location of the warp to a certain degree of approximation.

In the game Magicka (Arrowhead Game Studios 2011) two different kinds of teleports are available to the player. The spell 'Teleport' relocates the player's avatar to a location several meters in front of the start point. As the distance for this spell is always the same it is a predictable warp. The player knows to a certain degree where the avatar will end up if she uses the spell. The item 'Rod of Emergency Teleport' has an ability which also relocates the player. This emergency teleport relocates the player to a random location on the screen. Therefore, the 'Rod of Emergency Teleport' triggers an arbitrary relocation.

While single use warps have start and end points, limited use warps have at least one additional transitional point. Therefore, limited use warps could have a definite start, predictable transitional and arbitrary end location. Assuming a limited use warp with one transitional location, there are 21 combinations with different kinds of locations, some of which are more likely to occur in video games than others. However, a classification with 21 subcategories, and even more if we add multiple transitional locations, is unpractical. One possible solution for this is to classify a specific limited use warp by its lowest level of predictability. In the end, this is what has been done by calling a single use warp with an arbitrary end location an arbitrary warp, even though its start location is definite. A limited warp with definite start and end locations, but an arbitrary transitional location is, ultimately, an arbitrary limited use warp. If a more detailed analysis of specific warps is necessary, this system still provides the possibility of describing each location on its own, while the 'lowest level of predictability' approach enables a more convenient communication.

While the distinction between definite, predictable and arbitrary locations can be made for permanent use warps as well, they usually have definite start and end locations. In the first-person puzzle game The Talos Principle, the game's three main levels and 21 sublevels are connected through circles of light. These circles are connected in pairs and they exist throughout the whole play-through, which makes them a perfect example of definite permanent use relocations in geometrical space.

\section{The typology's application}

The typology enables us to classify specific navigational acts in a rigorous manner. This also means that its application is not primarily intended towards games as a whole. To apply it to a game would rather mean to examine all navigational acts in the game. 
This can of course yield interesting insights as well. However, for now the model will be applied to seven examples. Three examples will be discussed in more detail. Due to the scope of the paper, the others will just be mentioned in image 3 .

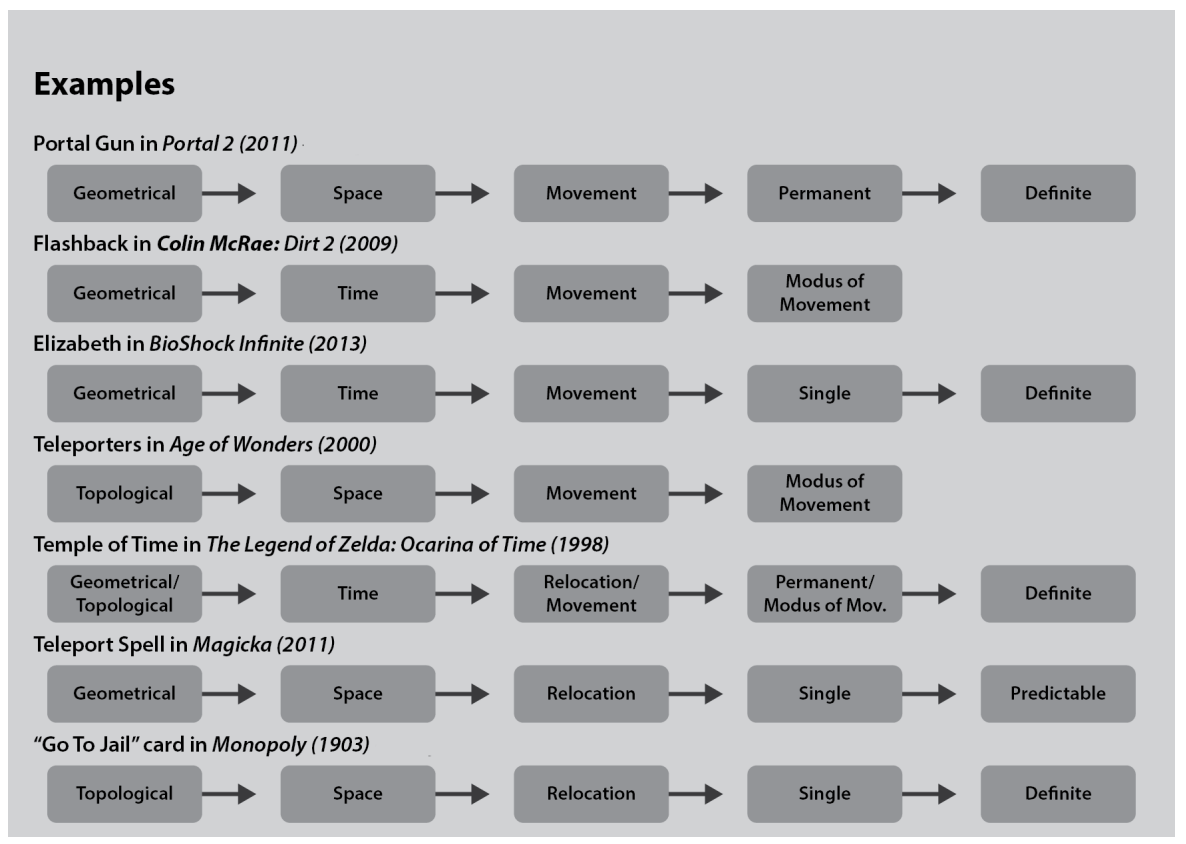

Figure 3. Examples for application ${ }^{1}$

The portal gun in Portal 2 creates an entrance and an exit on certain walls in the game. The player can move freely through these holes in a continuous manner. As the game occurs in a three dimensional, continuous space, the navigational acts overall category is geometrical. Furthermore, the gun creates holes that enable the player to move between originally distant, spatial locations, thus the classification as navigational act in geometrical space. The wormhole-like pathway for the player is navigable continuously. Therefore, this navigational act has to be understood as a tunnel that bends the game space, as opposed to a relocation, which is a discrete navigation. These portals are permanent tunnels, as they are available as many times as the player likes. Finally, the tunnel's start and end locations are

1 The BioShock Infinite (Irrational games 2013) example refers to a specific situation in which Elizabeth lifts Booker up a ledge, while simultaneously moving him through a temporal tunnel. 
specified by the player (or in special occasions the game) and therefore the portals in Portal 2 are definite permanent use tunnels in geometrical space.

Earlier in this paper a distinction between the prescribed plot and story was made. In Zelda we have to distinguish between the game's prescribed story as the game world's history, including all events that happened even if not presented to the player, and the prescribed plot as two different times (Link as a child and a young adolescent) that are available to the player, as topological organization of the game's time. Depending on whether the analysis aims for the understanding of the Citadel of Time in the game's prescribed story or plot, the navigational act has to be classified as navigation in geometrical or topological time categories respectively. All further classification relies on this distinction.

To start with the navigation in topological time, there are only these two discrete time zones available to the player. Therefore, drawing the Mastersword or putting it back in its place triggers a move in topological time and the classification ends here as a modus of movement between the prescribed plot's two discrete and adjacent areas. Considering the Citadel of Time in the frame of the game's geometrical history, the navigational act relocates Link to a far future or past, skipping all in-between events, making it a relocation in the game's prescribed story. Furthermore, the Citadel is always available to the player (after activation) and the temporal start and end points never change. Due to this we ultimately arrive at the Citadel's classification as a definite permanent use relocation in geometrical time.

An interesting case are the teleporters in Age of Wonders (Triumph Studios \& Epic MegaGames 2000). In this game space is organized topologically, giving the player only the choice between discrete locations. In topological environments, all navigation, whether relocation or movement, is discrete (see Günzel 2010, p. 174). Due to this the teleporters are - from a navigational perspective - not relocations, but actually moves. As the teleporters connect two 'distant' locations permanently and movement in topological space is always discrete, the locations of the teleporters are in fact adjacent. Following the definition of movement as transitioning between adjacent locations, units in Age of Wonders are merely moving, not relocating, between the teleporter locations ${ }^{2}$. Following the teleporter's classification

2 Here the teleporters are different from the 'Go to jail' card in Monopoly (Magie 1903). This is a single time occurrence, not a permanent connection of two locations, and thereby a single use relocation in topological space with definite end and predictable start location (it is impossible to know when the card will be drawn, but the start locations are limited to certain fields). 
as a move, the analysis of the teleporters ends here as a modus of movement in topological space.

This analysis shows that the present model can serve as the basis for new observations and discussions. First of all, the teleporters in Age of Wonders would be classified as relocations on a first glance, but through this more sophisticated examination from a navigational point of view, we have to ask the question if that initial observation can stand, or if we have to rethink our concepts of game space completely. Second of all, it also shows that we could - and maybe should - further distinguish between the visual and mechanical layers of navigation in videogames. While the units in Age of Wonders are mechanically only in one hexagon at a time, visually they do in fact traverse the game space in a continuous manner. This distinction can be useful for future investigations into the player's perception and experience of navigating videogame spaces.

\section{Conclusion}

This paper has dealt with the problem of an indistinct terminology of navigational acts. An overview of important research of space and time in game studies, as well as related work from other fields, has been given and was adapted. Through this theoretical work and the presented examples of video games, it was possible to develop exclusive categories for navigational acts.

The developed step-by-step classification of navigation in video games can be directly used for analyzing certain video game's navigational acts, or as a tool for comparing navigational acts across games. Such an application adds more depth to examinations of space and time in video games by putting an emphasis on the nature of navigational options given to the players inside these environments. This is important as it has been argued that the experience of a certain virtual environment can change, depending on how we navigate within it. In addition to this practical application during studies of games, the typology's development has delivered a terminology for a more sophisticated and standardized discussion of a multitude of aspects of video games and has already raised additional questions of videogame spatiality and analytical methods, through its alternative perspective. 
This research has received funding from the European Research Council (ERC) under the European Union's Horizon 2020 research and innovation programme (Grant Agreement No [695528] - Making Sense of Games). I also want to thank my anonymous reviewers for the valuable feedback, as well as Vinicius Rovtar Pinton for the creation of the images.

\section{References}

Aarseth, E. (1997). Cybertext: Perspectives on ergodic literature. Baltimore: JHU Press. Aarseth, E., Smedstad, S. M., \& Sunnanå, L. (2003). "A multi-dimensional typology of games". In M. Copier \& J. Raessens (eds.), Level Up: Digital Games Research Conference Proceedings. Utrecht, the Netherlands: Universteit Utrecht.

Aarseth, E. (2014). “Ontology”. In Wolf M. J. P., Perron, B. (eds.) The Routledge Companion to Video game studies, 484-492. New York: Routledge.

Bordwell, D., \& Thompson, K. (2008). Film Art. An Introduction. $8^{\text {th }}$ ed. Boston: McGraw Hill.

Caillois, R. (1961). Man, play, and games. Champaign: University of Illinois Press.

Calleja, G. (2009). "Experiential narrative in game environments". In Breaking New Ground: Innovation in Games, Play, Practice and Theory. Proceedings of DiGRA 2009.

Chesher, C. (2012). Navigating sociotechnical spaces: Comparing computer games and sat navs as digital spatial media. Convergence, 18(3), 315-330.

Deleuze, G., Guattari, F., \& Massumi, B. (trans.) (1987). A thousand plateaus: Capitalism and schizophrenia. Minneapolis: University of Minnesota Press.

Driel, L. Van, \& Bidarra, R. (2009). A semantic navigation model for video games. In International Workshop on Motion in Games (pp. 146-157). Springer Berlin Heidelberg.

Elverdam, C., \& Aarseth, E. (2007). “Game Classification and Game Design Construction Through Critical Analysis". In Games and Culture, 2(1), 3-22.

Eskelinen, M. (2001). The gaming situation. In Game studies, 1(1), 68.

Flynn, B. (2003). Languages of navigation within computer games. In Proceedings of MelbourneDAC, the 5th International Digital Arts and Culture Conference, 5-9.

Flynn, B. (2008). “The Navigator's Experience: An Examination of the Spatial in Computer Games". In Swalwell, M. and Wilson, J. (eds.) The Pleasures of Computer 
Gaming: Essays on Cultural History, Theory and Aesthetics, 119-143. Jefferson, NC: McFarland.

Gazzard, A. (2009a). Paths, players, places: Towards an understanding of mazes and spaces in videogames (Doctoral dissertation, University of Hertfordshire).

Gazzard, A. (2009b). "Teleporters, tunnels \& time: Understanding warp devices in videogames", paper presented to the conference Breaking New Ground: Innovation in Games, Play, Practice and Theory. Proceedings of DiGRA 2009.

Günzel, S. (2010). "The Space-Image". In Interactivity and Spatiality of Computer Games. Conference Proceedings of the Philosophy of Computer Games.

Nitsche, M. (2007). "Mapping time in video games". In Situated Play, Proceedings of DiGRA2007 Conference, 145-151.

Nitsche, M. (2008). Video game spaces: image, play, and structure in 3D game worlds. Cambridge: MIT Press.

Tychsen, A., \& Hitchens, M. (2009). "Game Time Modeling and Analyzing Time in Multiplayer and Massively Multiplayer Games". In Games and Culture, 4(2), 170-201.

Zagal, J. P., \& Mateas, M. (2007). "Temporal frames: a unifying framework for the analysis of game temporality". In Situated Play, Proceedings of DiGRA 2007 Conference, 516-522.

Ludography

Arrowhead Game Studios (2011). Magicka [PC]. Paradox Interactive.

Blizzard Entertainment (2012). Diablo 3 [PC]. Blizzard Entertainment: played June 2015.

Blizzard Entertainment (2015). Heroes of the Storm [PC]. Blizzard Entertainment: played June 2015.

Codemasters (2009). Colin McRae: Dirt 2 [PC]. Codemasters.

Croteam (2014). The Talos Principle [PC]. Devolver Digital.

Cyan (1993). Myst [Mac OS]. Brøderbund.

Dimps (2015). Dragon Ball XenoVerse [PC]. Bandai Namco Games.

HAL Laboratory (1999). Super Smash Bros. [Nintendo 64]. Nintendo.

Irrational Games (2013). BioShock Infinite [PC]. 2k Games.

Magie, Elisabeth (1935 [1903]). Monopoly [Board Game]. Parker Brothers, Hasbro.

Nintendo R\&D4 (1985). Super Mario Bros. [NES]. Nintendo.

Nintendo EAD (1998). The Legend of Zelda: Ocarina of Time [Nintendo 64]. Nintendo.

Runic Games (2012). Torchlight II [PC]. Runic Games. 
Triumph Studios, Epic MegaGames (2000). Age of Wonders. Gathering of Developers. Valve (1998). Half Life [PC]. Valve Corporation.

Valve Corporation (2000). Counter-Strike [PC]. Valve Corporation.

Valve Corporation (2011). Portal 2 [PC]. Valve Corporation.

Summary

Navigation in video games has been a vastly neglected topic in game studies. In this paper a classification system for navigational acts has been developed through theoretical work as well as the analysis of multiple games. The result is an exclusive five-step classification system. Moreover, the development showed that navigational acts are highly dependent on the environment in which they occur. The system is a first step towards a deeper understanding of how the player navigates the gameworld, instead of what she navigates.

Keywords: videogame space, videogame time, game ontology, navigation, typology

Michael S. Debus is a PhD Fellow in the ERC Advanced Grant funded MSG - Making Sense of Games project at the IT University of Copenhagen, Denmark. He holds an M.Sc. in Game Analysis from the same institution and a B.A. in Media Studies from the University of Siegen, Germany. 